\title{
Modeling Reliability Engineering Data Using Scale-Invariant Quasi-Inverse Lindley Model
}

\author{
Mohamed Kayid* and Tareq Alsayed \\ Department of Statistics and Operations Research, College of Science, King Saud University, Riyadh, Saudi Arabia \\ *Corresponding Author: Mohamed Kayid. Email: drkayid@ksu.edu.sa \\ Received: 22 November 2021; Accepted: 17 January 2022
}

\begin{abstract}
An important property that any lifetime model should satisfy is scale invariance. In this paper, a new scale-invariant quasi-inverse Lindley (QIL) model is presented and studied. Its basic properties, including moments, quantiles, skewness, kurtosis, and Lorenz curve, have been investigated. In addition, the well-known dynamic reliability measures, such as failure rate (FR), reversed failure rate (RFR), mean residual life (MRL), mean inactivity time (MIT), quantile residual life (QRL), and quantile inactivity time (QIT) are discussed. The FR function considers the decreasing or upside-down bathtub-shaped, and the MRL and median residual lifetime may have a bathtub-shaped form. The parameters of the model are estimated by applying the maximum likelihood method and the expectation-maximization (EM) algorithm. The EM algorithm is an iterative method suitable for models with a latent variable, for example, when we have mixture or competing risk models. A simulation study is then conducted to examine the consistency and efficiency of the estimators and compare them. The simulation study shows that the EM approach provides a better estimation of the parameters. Finally, the proposed model is fitted to a reliability engineering data set along with some alternatives. The Akaike information criterion (AIC), KolmogorovSmirnov (K-S), Cramer-von Mises (CVM), and Anderson Darling (AD) statistics are used to compare the considered models.
\end{abstract}

Keywords: Inverse Lindley distribution; reliability measures; maximum likelihood estimation; EM algorithm

\section{Introduction}

Lindley [1] and inverse Lindley models have attracted much attention in the last decade. There is a long list of research on the Lindley model and its generalizations. Ghitany et al. [2] studied some features of the Lindley model. Sankaran [3] applied the Lindley model to define a compound PoissonLindley distribution. Ghitany et al. [4] considered the distribution introduced by Sankaran [3] to study a compound Poisson-Lindley model truncated to zero. Zamani et al. [5] proposed and studied a compound negative binomial Lindley model. Ghitany et al. [6] considered a power Lindley model with two parameters. Al-Mutairi et al. [7] estimated the probability of stress strength for two independent

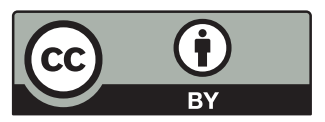

This work is licensed under a Creative Commons Attribution 4.0 International License, which permits unrestricted use, distribution, and reproduction in any medium, provided the original work is properly cited. 
Lindley distributions. Al-babtain et al. [8] generalized the Lindley model to a distribution with five parameters. Shanker et al. $[9,10]$ introduced an extended version of the Lindley model. Shanker et al. [11] investigated some mathematical properties of the extended Lindley model defined by Shanker et al. [10]. Moreover, Shanker et al. [12] introduced and studied a new quasi-Lindley model. Merovci et al. [13] proposed a beta Lindley model and studied its properties. Zakerzade et al. [14], Ibrahim et al. [15], and Shanker et al. [16] defined generalizations of the Lindley distribution with three parameters. Moreover, Broderick et al. [17] proposed a generalization of the Lindley model with four parameters.

Sharma et al. [18] introduced the inverse Lindley distribution and considered it as a stress-strength model. The probability density function (PDF) of the inverse Lindley distribution is

$f(x)=\frac{\theta^{2}}{1+\theta}\left(\frac{1+x}{x^{3}}\right) e^{-\frac{\theta}{x}}, \quad x>0, \theta>0$,

and follows an upside-down bathtub (unimodal) hazard rate function, so it is useful when the data has a unimodal hazard rate. Sharma et al. [19] have presented some data examples that follow models with such a hazard rate function. Alkarni [20] and Sharma et al. [19] proposed an extension of the inverse Lindley distribution with three parameters and a new generalized inverse Lindley distribution, respectively. Also, Barco et al. [21] have obtained a new distribution from the power Lindley distribution and the inverse Lindley distribution. Recently, Eltehiwy [22] studied the logarithmic transformation of the inverse Lindley distribution.

An important property that any lifetime model should satisfy is scale invariance. A distribution family with scale parameter $\theta$ and $\operatorname{PDF} f_{\theta}(x)$ is scale invariant if the change from $x$ to $k x$, $k>0$, does not change the family. More precisely, $f_{\theta}(k x)=J f_{\theta^{\prime}}(x)$ where $J$ is the Jacobian of the transformation. Unfortunately, the inverse Lindley and the generalized versions mentioned above are not scale invariant. In this paper, we therefore present and study a scale-invariant extension of the inverse Lindley distribution.

The rest of the paper is organized as follows. In Section 2, we introduce the new quasi-inverse Lindley model and study some of its properties. In Section 3, we apply maximum likelihood and EM to estimate the parameters of the model. In Section 4, we investigate the behaviour of the estimators through a simulation study. In Section 5, we fit the proposed model to a reliability engineering data set to show its applicability. Finally, we conclude the paper in Section 6.

\section{Quasi Inverse Lindley Distribution}

The scale invariant quasi inverse Lindley distribution, $\operatorname{QIL}(\alpha, \theta)$, is defined by the cumulative distribution function $(\mathrm{CDF})$

$F(x)=\frac{1}{\alpha+1}\left(\alpha+1+\frac{\theta}{x}\right) e^{-\frac{\theta}{x}}, \quad \alpha>0, \theta>0, x>0$.

The PDF of QIL is

$f(x)=\frac{\theta^{2}}{\alpha+1}\left(\frac{\alpha}{\theta} \frac{1}{x^{2}}+\frac{1}{x^{3}}\right) e^{-\frac{\theta}{x}}, \quad \alpha>0, \theta>0, x>0$.

It can be checked by differentiation that the sign of the $f^{\prime}(x)$ is equal with the sign of $-2 \frac{\alpha}{\theta} x^{2}+$ $(\alpha-3) x+\theta$. Thus $f^{\prime}(0)>0$ and $f$ increases at an early interval and then decreases, i.e., the PDF is unimodal for all values of the parameters. The PDF of the inverse gamma (IG) distribution with 
parameters $(v, \theta), I G(v, \theta)$, is of the form

$g(x)=\frac{\theta^{v}}{\Gamma(v)} x^{-v-1} e^{-\frac{\theta}{x}}, \quad v>0, \theta>0, x>0$,

so the PDF of QIL is a mixture of the PDF of inverse gamma distributions $I G(1, \theta)$ and $I G(2, \theta)$ with weights $\frac{\alpha}{\alpha+1}$ and $\frac{1}{\alpha+1}$. The QIL is an scale invariant extension of the the inverse Lindley distribution investigated by Sharma et al. [18], see scale invariance property discusssed in the Introduction section. If $\alpha$ be replaced by $\theta$ this new model will reduce to inverse Lindley. Moreover, if a random variable $X$ follows the QIL, then its reciprocal follows the model proposed by Shanker et al. [11].

Proposition 1. For $k<1$, the $k^{\text {th }}$ moment of the $Q I L(\alpha, \theta)$ is finite and equals

$E\left(X^{k}\right)=\frac{\theta^{k}}{\alpha+1}(\alpha \Gamma(1-k)+\Gamma(2-k))$,

while for $k \geq 1$ it is infinite.

Proof: The $k^{\text {th }}$ moment of the $\operatorname{QIL}(\alpha, \theta)$ is

$E\left(X^{k}\right)=\frac{\theta^{2}}{\alpha+1}\left(\int_{0}^{\infty} \frac{\alpha}{\theta} x^{k-2} e^{-\frac{\theta}{x}} d x+\int_{0}^{\infty} x^{k-3} e^{-\frac{\theta}{x}} d x\right)$.

The first integral of (6) simplifies to

$I_{1}=\int_{0}^{\infty} \frac{\alpha}{\theta} x^{k-2} e^{-\frac{\theta}{x}} d x=\alpha \theta^{k-2} \int_{0}^{\infty} z^{-k} e^{-z} d z$,

for $k<1$ equal to $\alpha \theta^{k-2} \Gamma(1-k)$. But, for $k=1, I_{1}$ reduces to

$$
\begin{aligned}
\frac{\alpha}{\theta} \int_{0}^{\infty} z^{-1} e^{-z} d z & =\frac{\alpha}{\theta}\left(\int_{0}^{a} z^{-1} e^{-z} d z+\int_{a}^{\infty} z^{-1} e^{-z} d z\right) \\
& >\frac{\alpha}{\theta} \int_{0}^{a} z^{-1} e^{-z} d z>\frac{\alpha}{\theta} e^{-a} \int_{0}^{a} z^{-1} d z=+\infty,
\end{aligned}
$$

in which arbitrary $a>0$. It shows that for $k \geq 1, E\left(X^{k}\right)$ is infinite. On the other hand, the second integral of (6) is

$\int_{0}^{\infty} x^{k-3} e^{-\frac{\theta}{x}} d x=\theta^{k-2} \Gamma(2-k)$.

Thus by (6) the proof is completed.

As a result of Proposition 1, and by the fact that

$e^{t X}=\sum_{k=0}^{\infty} \frac{t^{k} X^{k}}{k !}$

it follows that the moment generating function is infinite.

The quantile function $q(p)$ is defined by the inverse of the CDF. The quantile function has not a closed form for QIL and should be computed through the following

$(\alpha+1) p=\left(\alpha+1+\frac{\theta}{q(p)}\right) e^{-\frac{\theta}{q(p)}}$. 
The quantile function $q(p)$ can be used to describe distribution characteristics, especially skewness kurtosis and to estimate parameters. The skewness of the $Q I L$ can be measured by

$B=\frac{q(1-u)+q(u)-2 q(0.50)}{q(1-u)-q(u)}$,

where $u \in(0,0.5)$, (see MacGillivray [23]). The special case $u=0.25$ is referred to Bowley's measure of skewness (Bowley [24]). Also, the kurtosis of QIL is measured by (see Moors [25])

$K=\frac{q\left(\frac{7}{8}\right)-q\left(\frac{5}{8}\right)+q\left(\frac{3}{8}\right)-q\left(\frac{1}{8}\right)}{q\left(\frac{6}{8}\right)-q\left(\frac{2}{8}\right)}$.

The well-known Lorenz curve is a graphical representation for the inequality of distribution of wealth or income. It measures the proportion of overall wealth or income of the bottom $p$ percent of the people. The line of perfect equality is represented by the straight line between $(0,0)$ and $(1,1)$. The Lorenz curve is also a curve connecting these points and lies below the perfect equality line, see Bishop et al. [26]. For a model with the CDF $F$, the Lorenz curve is defined by

$L(p)=\frac{p \mu_{p}}{\mu}=\frac{\int_{0}^{q(p)} x d F(x)}{\int_{0}^{q(1)} x d F(x)}$.

For QIL, due to the fact that $\mu$ is infinite, but $\mu_{p}$ is finite for every $p$ such that $q(p)<\infty$, the Lorenz curve is zero and gives no information about income inequality. Fortunately, Prendergast et al. [27] defined three quantile versions of the Lorenz curve that can be applied to QIL. The main idea is to replace $\mu$ by median of the distribution and $\mu_{p}$ by its alternative quantile, i.e., $q\left(\frac{p}{2}\right)$. So, their alternatives for Lorenz curve are as follows.

$L_{1}(p)=p \frac{q\left(\frac{p}{2}\right)}{q(0.5)}$,

$L_{2}(p)=p \frac{q\left(\frac{p}{2}\right)}{q\left(1-\frac{p}{2}\right)}$,

and

$L_{3}(p)=2 p \frac{q\left(\frac{p}{2}\right)}{q\left(\frac{p}{2}\right)+q\left(1-\frac{p}{2}\right)}$.

\section{Dynamic Measures}

The FR, RFR, MRL, MIT, $p$-QRL and $p$-QIT play key role in the reliability and survival analysis. Let the reliability function of QIL be $R(x)=1-F(x)$, then the FR $\lambda(x)$ and RFR $\eta(x)$ functions of QIL are respectively,

$\lambda(x)=\frac{f(x)}{R(x)}=\frac{\theta^{2}\left(\frac{\alpha}{\theta} x+1\right)}{(\alpha+1) x^{3}\left(\exp \left(\frac{\theta}{x}\right)-1\right)+\theta x^{2}}, \quad x>0$,

and

$\eta(x)=\frac{f(x)}{F(x)}=\frac{\theta^{2}\left(\frac{\alpha}{\theta} x+1\right)}{(\alpha+1) x^{3}-\theta x^{2}}, \quad x>0$.

The FR function $\lambda(x)$ tends to zero when $x$ tends to zero or infinity. By differentiating from $\lambda(x)$ with respect to $x$, we find that the FR function shows a unimodal form. The RFR function $\eta(x)$ tends to infinity at zero and tends to zero at infinity and is a decreasing function, refer to Lai et al. [28]. 
The MRL function $m(x)$ represents the expectation of the conditional remaining life of an object given that it has been survived up to time $x$ and equals $m(x)=E(X-x \mid X \geq x)$, see Lai et al. [28].

Proposition 2. The MRL function $m(x)$ of the QIL is infinite for $x \geq 0$.

Proof: The MRL can be expressed by

$m(x)=\frac{1}{\bar{F}(x)} \int_{x}^{\infty} t f(t) d t-x$

With straightforward algebra we have

$\int_{x}^{\infty} t f(t) d t=\frac{\theta^{2}}{\alpha+1}\left(\int_{x}^{\infty} \frac{\alpha}{\theta} t^{-1} \exp \left(-\frac{\theta}{t}\right) d t+\int_{x}^{\infty} t^{-2} \exp \left(-\frac{\theta}{t}\right) d t\right)$.

The first integral (8) is not finite since

$\int_{x}^{\infty} \frac{\alpha}{\theta} t^{-1} \exp \left(-\frac{\theta}{t}\right) d t=\frac{\alpha}{\theta} \int_{0}^{\frac{\theta}{x}} \frac{1}{z} e^{-z} d z>\frac{\alpha}{\theta} e^{-\frac{\theta}{x}} \int_{0}^{\frac{\theta}{x}} \frac{1}{z} d z=+\infty$,

which shows the proposition.

The MIT $v(x)$ describes the conditional expectation of the elapsed time of an event given that it has been happened sometime before $x$, more formally $v(x)=E(x-X \mid X<x)$, see Lai et al. [28]. The following proposition shows that the MIT of QIL is finite for all $x>0$.

Proposition 3. The MIT is finite for all $x>0$ and

$v(x)=x-\frac{1}{F(x)}\left(\frac{\theta^{2}}{\alpha+1}\left(\frac{\alpha}{\theta} \int_{\frac{\theta}{x}}^{\infty} \frac{1}{z} e^{-z} d z+\frac{1}{\theta} e^{-\frac{\theta}{x}}\right)\right)$.

Proof: The MIT can be written as

$v(x)=x-\frac{1}{F(x)} \int_{0}^{x} t f(t) d t, \quad x>0$,

and for the QIL, we have

$\int_{0}^{x} t f(t) d t=\frac{\theta^{2}}{\alpha+1}\left(\int_{0}^{x} \frac{\alpha}{\theta} t^{-1} \exp \left(-\frac{\theta}{t}\right) d t+\int_{0}^{x} t^{-2} \exp \left(-\frac{\theta}{t}\right) d t\right)$.

The first integral (10) can be simplified to

$\int_{0}^{x} \frac{\alpha}{\theta} t^{-1} \exp \left(-\frac{\theta}{t}\right) d t=\frac{\alpha}{\theta} \int_{\frac{\theta}{x}}^{\infty} z^{-1} e^{-z} d z<\frac{\alpha}{\theta} \int_{\frac{\theta}{x}}^{\infty} z^{-2} d z<\infty$.

The second integral of $(10)$ reduces to

$\int_{0}^{x} t^{-2} \exp \left(-\frac{\theta}{t}\right) d t=\frac{1}{\theta} \exp \left(-\frac{\theta}{x}\right)$,

which completes the proof.

The $p$-QRL function, denoted by $q_{p}(x)$, represents the conditional $p$ th quantile of the remaining life of an object, given that it has been survived up to time $x$,

$q_{p}(x)=\bar{F}^{-1}(\bar{p} \bar{F}(x))-x=q(1-\bar{p} \bar{F}(x))-x, \quad x>0$,

where the quantile function $q$ is defined in (7) and $\bar{p}=1-p$. 
The $p$-QIT $q_{p}^{*}(x)$, represents the conditional $p$ th quantile of the elapsed time of an event, given that it has been happened sometime before $x$, more formally

$q_{p}^{*}(x)=x-F^{-1}(\bar{p} F(x))=x-q(\bar{p} F(x)), \quad x>0$.

Similar to the quantile function $q$ defined by (7), the $p$-QRL and $p$-QIT have not closed form for QIL. Thus, we should be computed them numerically. When $p=0.5$, the $p$-QRL and $p$-QIT are known as the median residual life and the median inactivity time respectively.

Fig. 1 draws the density and FR functions of QIL for some parameters. The density is more skewed to right for larger $\theta$. The FR shows upside down bathtub shape (unimodal form).
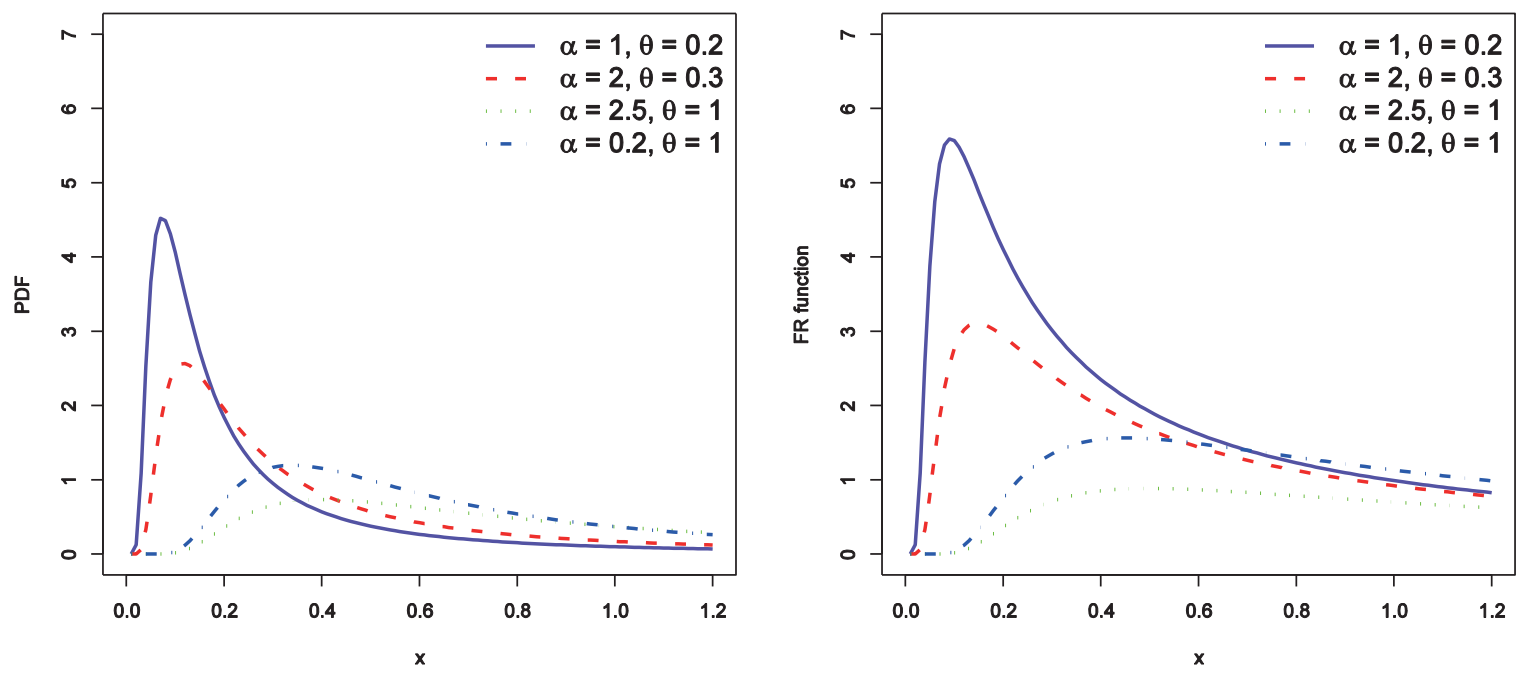

Figure 1: The PDF and FR function of $\operatorname{QIL}(\alpha, \theta)$ for some values of parameters

\section{Estimation of the Parameters}

In this section, we discuss the maximum likelihood estimation (MLE) method and the EM algorithm for estimating the parameters of the proposed model (1).

\subsection{The $M L E$}

Let $x_{1}, x_{2}, \ldots, x_{n}$ be an independent and identically distributed (iid) realization from $Q I L(\alpha, \theta)$, the log-likelihood function of the parameters is

$l(\alpha, \theta ; x)=2 n \ln (\theta)-n \ln (1+\alpha)+\sum_{i=1}^{n} \ln \left(\frac{\alpha}{\theta} \frac{1}{x_{i}^{2}}+\frac{1}{x_{i}^{3}}\right)-\theta \sum_{i=1}^{n} \frac{1}{x_{i}}$.

Then, the likelihood equations are

$\frac{\partial l}{\partial \alpha}=-\frac{n}{\alpha+1}+\sum_{i=1}^{n} \frac{x_{i}}{\alpha x_{i}+\theta}=0$, 
and

$\frac{\partial l}{\partial \theta}=\frac{2 n}{\theta}-\frac{1}{\theta} \sum_{i=1}^{n}\left(\frac{1}{\alpha} \frac{\theta}{x_{i}}+1\right)^{-1}-\sum_{i=1}^{n} \frac{1}{x_{i}}=0$.

The MLE can compute by maximizing the log-likelihood function directly, or solving the likelihood equations. The first approach has applied in the next sections.

Let $l=\ln f(X)$, then the following matrix shows the Fisher information.

$K=\left[\begin{array}{cc}E\left(-\frac{\partial^{2} l}{\partial \alpha^{2}}\right) & E\left(-\frac{\partial^{2} l}{\partial \alpha \partial \theta}\right) \\ E\left(-\frac{\partial^{2} l}{\partial \theta \partial \alpha}\right) & E\left(-\frac{\partial^{2} l}{\partial \theta^{2}}\right)\end{array}\right]$

The variance of the MLE $\operatorname{Var}(\hat{\alpha}, \hat{\theta})$ can be approximated by $n^{-1} K^{-1}$, where $K^{-1}$ is the inverse of the information matrix. Moreover, for an iid random sample $X_{i}, i=1,2, \ldots, n$ from $Q I L\left(\alpha_{0}, \theta_{0}\right)$ the asymptotic distribution of $\sqrt{n}\left(\hat{\alpha}-\alpha_{0}, \hat{\theta}-\theta_{0}\right)$ is normal with mean $\mathbf{0}$ and variance $K^{-1}$. The asymptotic distribution can be used for providing confidence intervals of the parameters or in hypothesis testing problems.

\subsection{The EM Algorithm}

As explained earlier, the $\operatorname{QIL}(\alpha, \theta)$ is a mixture of two inverse gamma distributions $\operatorname{IG}(1, \theta)$ and $I G(2, \theta)$ and $\alpha$ determines the weights of the mixture. Let $x_{i}$ be an instance of the QIL model. Imagine one unobserved latent random variable $Z_{i}$ which determines that $x_{i}$ comes from $I G(1, \theta)$ or $I G(2, \theta)$. Let $X_{i}, i=1,2, \ldots, n$ be an iid random sample, $X_{i} \mid Z_{i}=j \sim I G(j, \theta), j=1,2, P\left(Z_{i}=1\right)=\frac{\alpha}{\alpha+1}$ and $P\left(Z_{i}=2\right)=\frac{1}{\alpha+1}$. Then $X_{i}$ follows $Q I L(\alpha, \theta)$. For simplicity, let $\beta=(\alpha, \theta)$. The likelihood function is

$L(\beta ; \mathbf{x}, \mathbf{z})=\prod_{i=1}^{n} \prod_{j=1}^{2}\left(g_{j}\left(x_{i} ; \theta\right) P_{Z_{i}}(j)\right)^{I\left(z_{i}=j\right)}$,

where $I\left(z_{i}=j\right)$ equals 1 when $z_{i}=j$ and equals 0 otherwise. Also, $P_{Z_{i}}(j)=P\left(Z_{i}=j\right)=\frac{1}{\alpha+1} \alpha^{I(j=1)}$ and

$g_{j}\left(x_{i} ; \theta\right)=\theta^{j} x^{-j-1} \exp \left(-\frac{\theta}{x_{i}}\right)$

is the PDF of the $I G(j, \theta)$. Then the log-likelihood function can be simplified to the following form.

$l(\beta ; \mathbf{x}, \mathbf{z})=\ln L(\beta ; \mathbf{x}, \mathbf{z})=\sum_{i=1}^{n} \sum_{j=1}^{2} I\left(z_{i}=j\right) \ln \left(\theta^{j} x_{i}^{-j-1} \exp \left(-\frac{\theta}{x_{i}}\right) \frac{1}{\alpha+1} \alpha^{I(j=1)}\right)$. 
The following expectation (E) and maximization (M) steps:

\section{The E Step}

Given the estimate of the parameters at iteration $t, \beta_{t}$, the conditional distribution of $Z_{i}$ can compute by Bayes theorem as

$$
\begin{aligned}
p_{i j, t}=P\left(Z_{i}=j \mid X_{i}=x_{i}, \beta_{t}\right) & =\frac{f\left(X_{i}=x_{i} \mid Z_{i}=j, \beta_{t}\right) P\left(Z_{i}=j \mid \beta_{t}\right)}{f\left(X_{i}=x_{i} \mid \beta_{t}\right)} \\
& =\frac{\theta_{t}^{j} x_{i}^{-j-1} \exp \left(-\frac{\theta_{t}}{x_{i}}\right)\left(\alpha_{t}+1\right)^{-1} \alpha_{t}^{I(j=1)}}{\left(\theta_{t} x_{i}^{-2} \alpha_{t}+\theta_{t}^{2} x_{i}^{-3}\right)\left(\alpha_{t}+1\right)^{-1} \exp \left(-\frac{\theta_{t}}{x_{i}}\right)},
\end{aligned}
$$

which are known as membership probabilities at iteration $t$, and applied to obtain the expectation function $Q\left(\beta \mid \beta_{t}\right)$ as follows.

$$
\begin{aligned}
Q\left(\beta \mid \beta_{t}\right)= & E_{Z \mid X, \beta_{t}}(l(\beta ; \mathbf{x}, \mathbf{Z})) \\
= & \sum_{i=1}^{n} E_{Z_{i} \mid X_{i}, \beta_{t}} \sum_{j=1}^{2} I\left(z_{i}=j\right) \ln \left(\theta^{j} x_{i}^{-j-1} \exp \left(-\frac{\theta}{x_{i}}\right) \frac{1}{\alpha+1} \alpha^{I(j=1)}\right) \\
= & \sum_{i=1}^{n} P\left(Z_{i}=1 \mid X_{i}=x_{i}, \beta_{t}\right) \ln \left(\theta x_{i}^{-2} \exp \left(-\frac{\theta}{x_{i}}\right) \frac{\alpha}{\alpha+1}\right) \\
& +P\left(Z_{i}=2 \mid X_{i}=x_{i}, \beta_{t}\right) \ln \left(\theta^{2} x_{i}^{-3} \exp \left(-\frac{\theta}{x_{i}}\right) \frac{1}{\alpha+1}\right) \\
= & \sum_{i=1}^{n} p_{i 1, t} \ln \left(\theta x_{i}^{-2} \exp \left(-\frac{\theta}{x_{i}}\right) \frac{\alpha}{\alpha+1}\right)+p_{i 2, t} \ln \left(\theta^{2} x_{i}^{-3} \exp \left(-\frac{\theta}{x_{i}}\right) \frac{1}{\alpha+1}\right) \\
= & \sum_{i=1}^{n}\left(p_{i 1, t} \ln \alpha-\ln (\alpha+1)\right) \\
& +\sum_{i=1}^{n}\left(p_{i 1, t} \ln \left(\theta x_{i}^{-2} \exp \left(-\frac{\theta}{x_{i}}\right)\right)+p_{i 2, t} \ln \left(\theta^{2} x_{i}^{-3} \exp \left(-\frac{\theta}{x_{i}}\right)\right)\right) .
\end{aligned}
$$

Thus the expectation function can be arranged as a sum of two expressions which one expression just depends on $\alpha$ and the other term just depends on $\theta$.

$Q\left(\beta \mid \beta_{t}\right)=Q_{1}\left(\alpha \mid \beta_{t}\right)+Q_{2}\left(\theta \mid \beta_{t}\right)$,

where

$Q_{1}\left(\alpha \mid \beta_{t}\right)=\sum_{i=1}^{n}\left(p_{i 1, t} \ln \alpha-\ln (\alpha+1)\right)$

and

$Q_{2}\left(\theta \mid \beta_{t}\right)=\sum_{i=1}^{n}\left(p_{i 1, t} \ln \left(\theta x_{i}^{-2} \exp \left(-\frac{\theta}{x_{i}}\right)\right)+p_{i 2, t} \ln \left(\theta^{2} x_{i}^{-3} \exp \left(-\frac{\theta}{x_{i}}\right)\right)\right)$. 


\section{The M Step}

To find the estimation of the parameters at $t+1$ iteration, the objective expression $Q\left(\beta \mid \beta_{t}\right)$ should be maximized in terms of $\beta$. So, it results that

$\beta_{t+1}=\arg \max _{\beta} Q\left(\beta \mid \beta_{t}\right)$,

which by (18) can reduce to the two following separate maximization problems

$\alpha_{t+1}=\arg \max _{\alpha} Q_{1}\left(\alpha \mid \beta_{t}\right)$,

and

$\theta_{t+1}=\arg \max _{\theta} Q_{2}\left(\theta \mid \beta_{t}\right)$,

where $Q_{1}\left(\alpha \mid \beta_{t}\right)$ and $Q_{2}\left(\theta \mid \beta_{t}\right)$ are determined by (19) and (20) respectively and by differentiating from $Q_{1}\left(\alpha \mid \beta_{t}\right)$ and $Q_{2}\left(\theta \mid \beta_{t}\right)$ with respect to $\alpha$ and $\theta$ respectively, it follows that

$\alpha_{t+1}=\frac{\sum_{i=1}^{n} p_{i 1, t}}{n-\sum_{i=1}^{n} p_{i 1, t}}$

and

$\theta_{t+1}=\frac{\sum_{i=1}^{n}\left(p_{i 1, t}+2 p_{i 2, t}\right)}{\sum_{i=1}^{n} x_{i}^{-1}}$.

The iterative process can conclude for some predefined small $\epsilon>0$,

$Q\left(\beta_{t+1} \mid \beta_{t+1}\right)<Q\left(\beta_{t} \mid \beta_{t}\right)+\epsilon$.

\section{Simulation}

By a simulation study, the efficiency of the MLE and EM estimator have been investigated and compared. The fact that QIL is a mixture of two inverse gamma distribution to provide random samples. More specifically, the following steps should be performed:

- Simulate one sample of multinomial distribution with parameters $n, p=\frac{\alpha}{\alpha+1}$ and $1-p$. Let the generated instance be $n_{1}$ and $n_{2}$, corresponding to probabilities $p$ and $1-p$ respectively.

- Generate one sample with size $n_{1}$ from gamma distribution $G(1, \theta)$ and another sample from $G(2, \theta)$ with size $n_{2}$. Combine two generated samples to provide one sample of $Q I L(\alpha, \theta)$ with size $n$.

In each run, some suitable values for the parameters are selected. Then, $r=5000$ repetitions of random samples of QIL are simulated. The size of samples are $n=50,150$ or 250 . For each repetition, the parameters were estimated using the maximum likelihood method or the EM approach.

The function "nleqslv" of the library "Ineqslv" in R was used to calculate the MLE. This function solves the likelihood Eqs. (12) and (13) to find the MLE. The initial values were randomly generated by a uniform distribution in both the MLE and EM approaches. In the EM algorithm, checking the termination condition in each EM iteration causes the runs very slow. Therefore, the EM algorithm was tested many times to find out how many iterations are sufficient. We found that 5 iterations is sufficient. Tab. 1 shows the bias (B) and mean square error (MSE) of the computed estimators. In every cell of this table the first and second lines show B and MSE for $\alpha$ and $\theta$ respectively. Let $\hat{\alpha}$ 
represent the MLE of $\alpha$, then its corresponding B and MSE are defined by the following relations:

$B_{\hat{\alpha}}=\frac{1}{r} \sum_{i=1}^{r}\left(\hat{\alpha}_{i}-\alpha\right)$

and

$M S E_{\hat{\alpha}}=\frac{1}{r} \sum_{i=1}^{r}\left(\hat{\alpha}_{i}-\alpha\right)^{2}$.

Table 1: Simulation results for MLE and EM estimator of the parameters of QIL distribution. In every cell the first and second lines are related to $\alpha$ and $\theta$ respectively

\begin{tabular}{|c|c|c|c|c|c|c|c|}
\hline & \multirow[b]{3}{*}{$\alpha, \theta$} & \multicolumn{6}{|c|}{$n$} \\
\hline & & \multicolumn{2}{|l|}{50} & \multicolumn{2}{|c|}{150} & \multicolumn{2}{|c|}{250} \\
\hline & & B & MSE & B & MSE & B & MSE \\
\hline \multirow[t]{6}{*}{$\overline{M L E}$} & \multirow[t]{2}{*}{$1,0.02$} & 2.741332 & 31.83574 & 1.035581 & 8.269541 & 0.526770 & 3.365813 \\
\hline & & -0.001375 & 0.000016 & -0.000568 & 0.000007 & -0.000326 & 0.000005 \\
\hline & \multirow[t]{2}{*}{$2,0.1$} & 13.76979 & 460.7387 & 6.997355 & 143.9364 & 4.597406 & 74.97038 \\
\hline & & -0.011009 & 0.000439 & -0.00765 & 0.000263 & -0.005475 & 0.000193 \\
\hline & \multirow[t]{2}{*}{$0.7,0.5$} & 17.10285 & 1524.676 & 4.366955 & 329.8249 & 2.39857 & 178.3365 \\
\hline & & -0.035906 & 0.012870 & -0.011314 & 0.004737 & -0.006114 & 0.002749 \\
\hline \multirow[t]{6}{*}{ EM } & \multirow[t]{2}{*}{$1,0.02$} & 0.013899 & 0.059163 & 0.008365 & 0.027584 & 0.007138 & 0.021205 \\
\hline & & 0.000325 & 0.000005 & 0.000086 & 0.000002 & 0.000068 & 0.000001 \\
\hline & \multirow[t]{2}{*}{$2,0.1$} & 0.032409 & 0.206652 & 0.020397 & 0.107106 & 0.018286 & 0.087159 \\
\hline & & 0.001878 & 0.000150 & 0.000721 & 0.000052 & 0.000239 & 0.000031 \\
\hline & \multirow[t]{2}{*}{$0.7,0.5$} & 0.008342 & 0.032587 & 0.005476 & 0.014904 & 0.006162 & 0.011417 \\
\hline & & 0.005695 & 0.003174 & 0.001787 & 0.001062 & 0.001410 & 0.000665 \\
\hline
\end{tabular}

Other measurements are defined similarly. Some of the simulation results are listed in the following:

- As sample size increases, the MSE decrease, in both MLE and EM approaches, i.e., the MLE and EM estimators are consistent.

- The MSE of MLE shows unexpectedly large values especially for $\alpha$. Fortunately, the EM approach shows far smaller MSEs for both $\alpha$ and $\theta$. Thus, the EM outperforms MLE in terms of the MSE.

\section{Applications}

In this section, we fit the proposed model to a data set to show its applicability. Tab. 2 represents one data set consists of 46 observations reported on active repair times in terms of hours for an airborne communication transceiver discussed by Alven [29]. The $Q I L$ is fitted to this data set and the parameters have been estimated by the maximum likelihood method and EM algorithm. The 
computed MLE and EM estimation are $(\hat{\alpha}, \hat{\theta})=(14.997,1.2066)$ and $(\tilde{\alpha}, \tilde{\theta})=(19.6777,1.1911)$ respectively. In a comparative analysis, the IG, inverse Weibull (IW) and power inverse Lindley (PIL) distributions are fitted to this dataset. The PDF of the IG is defined by (4) and the PDF of the IW and PIL are respectively

$f(x)=\alpha \theta x^{-\theta-1} e^{-\alpha x^{-\theta}}, \quad \alpha>0, \theta>0, x \geq 0$,

and

$f(x)=\frac{\alpha \theta^{2}}{1+\theta} \frac{1+x^{\alpha}}{x^{2 \alpha+1}} e^{-\theta x^{-\alpha}}, \quad \alpha>0, \theta>0, x \geq 0$.

Table 2: Active repair times (hours) for an airborne communication transceiver

\begin{tabular}{lllllllllll}
\hline 0.2 & 0.3 & 0.5 & 0.5 & 0.5 & 0.5 & 0.6 & 0.6 & 0.7 & 0.7 & 0.7 \\
\hline 0.8 & 0.8 & 1.0 & 1.0 & 1.0 & 1.0 & 1.1 & 1.3 & 1.5 & 1.5 & 1.5 \\
1.5 & 2.0 & 2.0 & 2.2 & 2.5 & 2.7 & 3.0 & 3.0 & 3.3 & 3.3 & 4.0 \\
4.0 & 4.5 & 4.7 & 5.0 & 5.4 & 5.4 & 7.0 & 7.5 & 8.8 & 9.0 & 10.3 \\
22.0 & 24.5 & & & & & & & & & \\
\hline
\end{tabular}

The results of fitting models are abstracted in Tab. 3. The estimates of the parameters, AIC, K-S, CVM and A-D statistics are computed. The QIL model outperforms the other candidates in terms of the AIC, K-S, CVM and A-D statistics. Also, the great p-values (near one) indicates good and competitive fits for all models. The empirical and fitted CDFs of QIL along with the alternative models are drawn in Fig. 2. Also, the right side of Fig. 3 shows upside down bathtub shape for the FR function of all of the estimated models. The total time on test plot of the data set presented by Fig. 4 shows a plot which is above the identity line at the beginning and then falls below the identity line. Thus, Fig. 4 confirms an upside down bathtub shape for the FR function, too.

Table 3: The results of fitting the QIL model and some alternative models to dataset of Tab. 2

\begin{tabular}{|c|c|c|c|c|c|c|}
\hline Model & $\hat{\alpha}$ & $\hat{\theta}$ & AIC & $\mathrm{K}-\mathrm{S}$ p-V & CVM p-v & $A D p-v$ \\
\hline QIL (EM) & 19.6777 & 1.1911 & - & $\begin{array}{l}0.0765 \\
0.9506\end{array}$ & $\begin{array}{l}0.0474 \\
0.8938\end{array}$ & $\begin{array}{l}0.3478 \\
0.8979\end{array}$ \\
\hline QIL (MLE) & 14.997 & 1.2066 & 204.4159 & $\begin{array}{l}0.0769 \\
0.9481\end{array}$ & $\begin{array}{l}0.0478 \\
0.8914\end{array}$ & $\begin{array}{l}0.3493 \\
0.8965\end{array}$ \\
\hline IG & 1.0785 & 1.2254 & 205.231 & $\begin{array}{l}0.0919 \\
0.8319\end{array}$ & $\begin{array}{l}0.0636 \\
0.7932\end{array}$ & $\begin{array}{l}0.3980 \\
0.8499\end{array}$ \\
\hline IW & 2.1314 & 1.0128 & 205.3814 & $\begin{array}{l}0.0807 \\
0.9251\end{array}$ & $\begin{array}{l}0.0510 \\
0.8721\end{array}$ & $\begin{array}{l}0.3573 \\
0.8892\end{array}$ \\
\hline PIL & 0.9378 & 1.6022 & 205.9927 & $\begin{array}{l}0.0798 \\
0.9310\end{array}$ & $\begin{array}{l}0.0527 \\
0.8617\end{array}$ & $\begin{array}{l}0.3843 \\
0.8635\end{array}$ \\
\hline
\end{tabular}



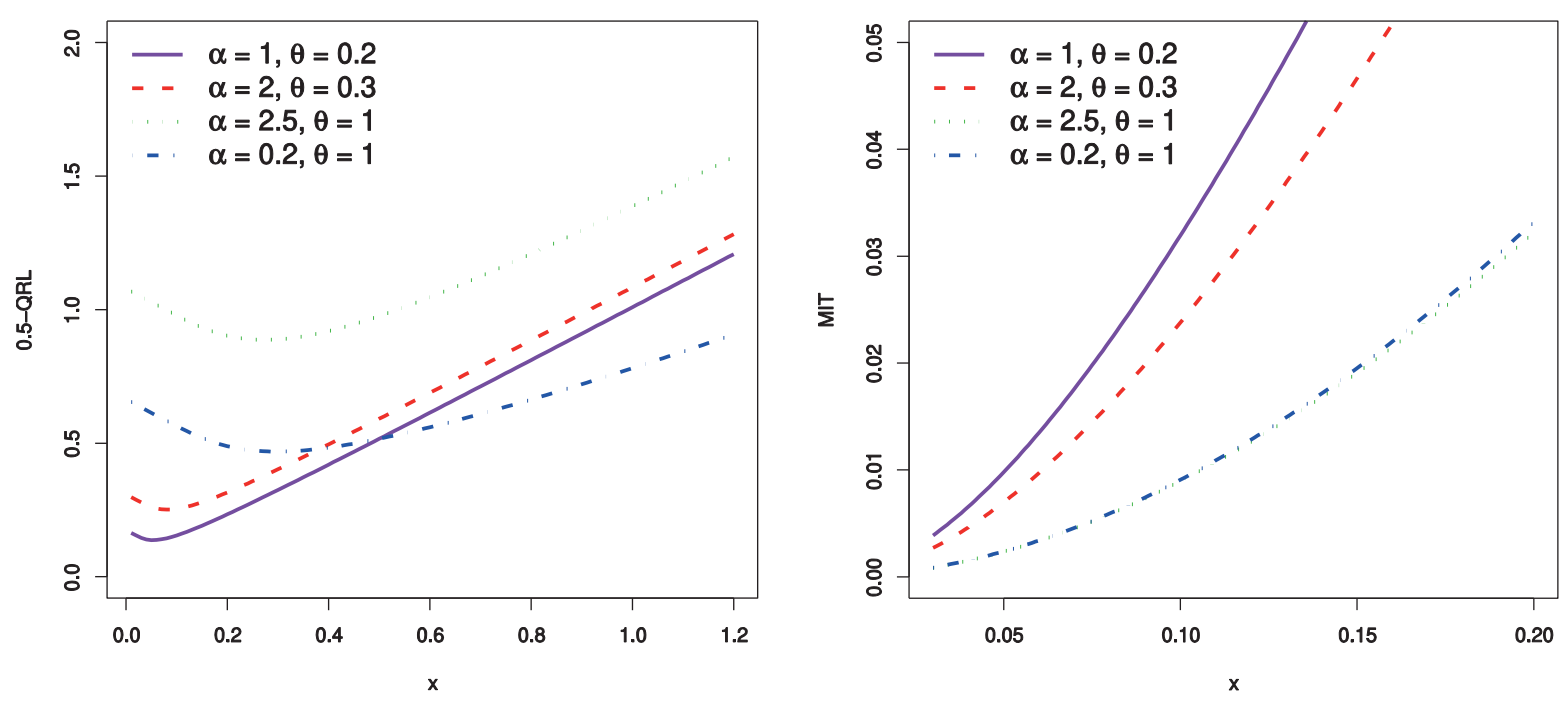

Figure 2: The 0.5-QRL function and the MIT function of $Q I L(\alpha, \theta)$ for some values of parameters
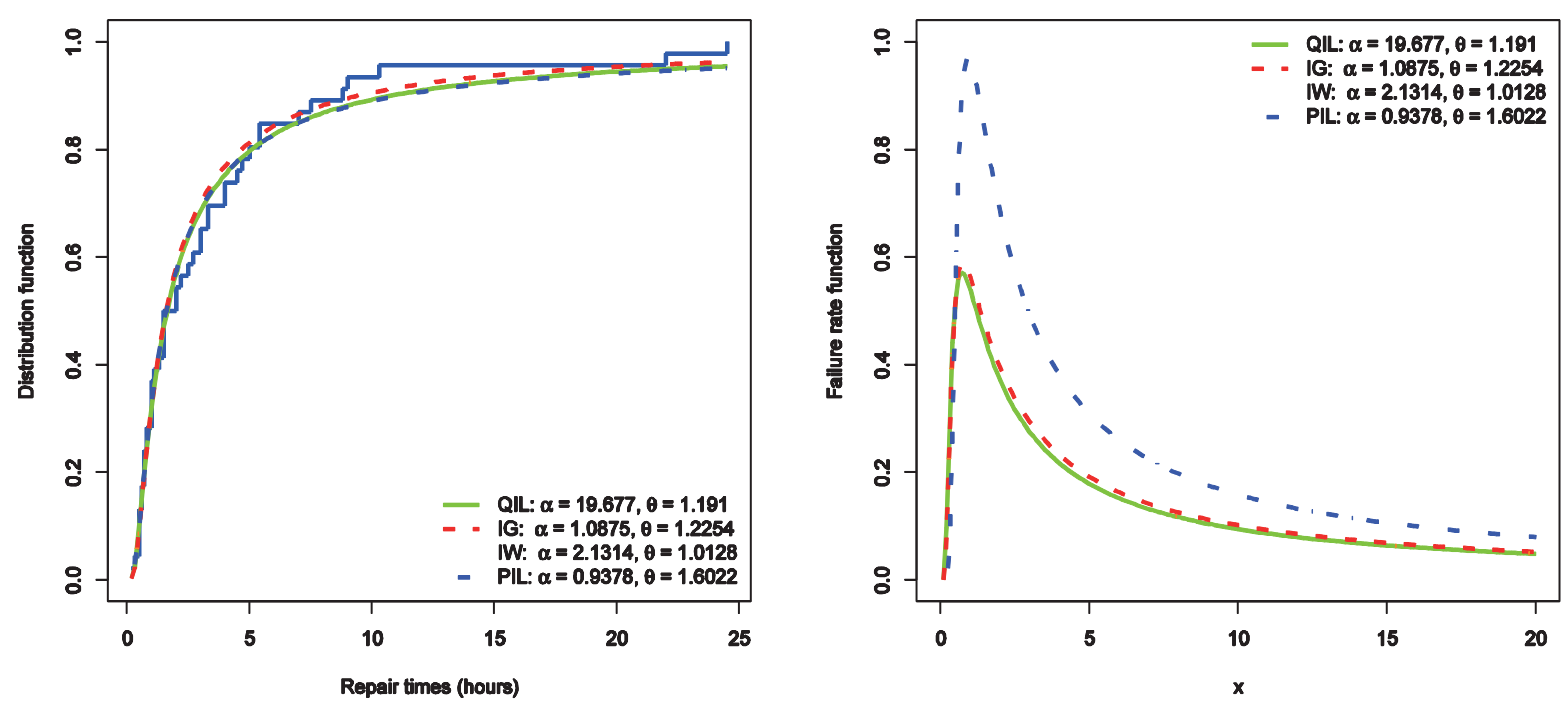

Figure 3: Left: The empirical distribution and fitted distributions to the data set of Tab. 2. Right: The FR function of the estimated distributions 


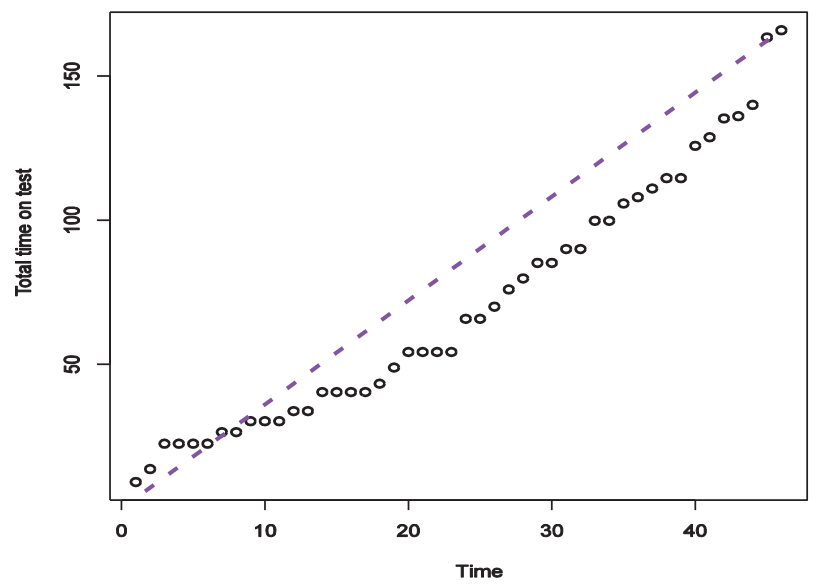

Figure 4: The total time on test plot for data set of Tab. 2. This plot reveals an upside-down bathtub shape form for the FR function

\section{Conclusions}

A new scale-invariant quasi-Lindley distribution was introduced and studied. It is useful for the analysis of lifetime data with an upside-down bathtub shape FR function. The elementary properties of the proposed model were explored. Also, some dynamic reliability measures were investigated. The maximum likelihood and EM methods were discussed. A simulation study was conducted to investigate and compare the behavior of the two approaches. It found that the EM method can estimate the parameters more efficiently.

Acknowledgement: The authors are grateful to anonymous referees for their constructive comments and suggestions, which has greatly improved this paper.

Funding Statement: This work is supported by Researchers Supporting Project Number (RSP2021/392), King Saud University, Riyadh, Saudi Arabia.

Conflicts of Interest: The authors declare that they have no conflicts of interest to report regarding the present study.

\section{References}

[1] D. V. Lindley, "Fiducial distributions and Bayes' theorem," Journal of the Royal Statistical Society. Series B (Methodological), vol. 20, pp. 102-107, 1958.

[2] M. E. Ghitany, B. Atieh and S. Nadarajah, "Lindley distribution and its application," Mathematics and Computers in Simulation, vol. 78, pp. 493-506, 2008.

[3] M. Sankaran, "The discrete poisson-lindley distribution," Biometrics, vol. 26, pp. 145-149, 1970.

[4] M. E. Ghitany, D. K. Al-Mutairi and S. Nadarajah, "Zero-truncated poisson-lindley distribution and its application," Mathematics and Computers in Simulation, vol. 79, pp. 279-287, 2008.

[5] H. Zamani and N. Ismail, "Negative binomial-lindley distribution and its application," Journal of Mathematics and Statistics, vol. 6, pp. 4-9, 2010.

[6] M. E. Ghitany, D. K. Al-Mutairi, N. Balakrishnan and L. J. Al-Enezi, "Power lindley distribution and associated inference," Computational Statistics and Data Analysis, vol. 64, pp. 20-33, 2013. 
[7] D. K. Al-Mutairi, M. E. Ghitany and D. Kundu, "Inferences on stress-strength reliability from lindley distribution," Communications in Statistics-Theory and Methods, vol. 42, pp. 1443-1463, 2013.

[8] A. A. Al-babtain, H. A. Eid, N. A. A-Hadi and F. Merovci, "The five parameter lindley distribution," Pakistan Journal of Statistics, vol. 31, pp. 363-384, 2014.

[9] R. Shanker and A. Mishra, "A quasi lindley distribution," African Journal of Mathematics and Computer Science. Research, vol. 6, pp. 64-71, 2013.

[10] R. Shanker and A. Mishra, "A two-parameter lindley distribution," Statistics in Transition-New Series, vol. 14, no. 1, pp. 45-56, 2013.

[11] R. Shanker, H. Fesshaye and S. Sharma, "On two-parameter lindley distribution and its applications to model lifetime data," Biometrics and Biostatistics International Journal, vol. 1, pp. 9-15, 2016.

[12] R. Shanker and A. H. Ghebretsadik, "A new quasi lindley distribution," International Journal of Statistics and Systems, vol. 8, pp. 143-156, 2013.

[13] F. Merovci and V. K. Sharma, "The beta-lindley distribution: Properties and applications," Journal of Applied Mathematics, vol. 2014, p. 10, Article ID 198951, 2014.

[14] H. Zakerzadeh and A. Dolati, "Generalized lindley distribution," Journal of Mathematical Extension, vol. 3, pp. 13-25, 2009.

[15] E. Ibrahim, F. Merovci and M. Elgarhy, "A new generalized lindley distribution," Mathematical Theory and Modeling, vol. 3, no. 13, pp. 30-47, 2013.

[16] R. Shanker, K. K. Shukla, R. Shanker and T. A. Leonida, "A three-parameter lindley distribution," American Journal of Mathematics and Statistics, vol. 7, no. 1, pp. 15-26, 2017.

[17] O. O. Broderick and Y. Tiantian, "A new class of generalized lindley distributions with applications," Journal of Statistical Computation and Simulation, vol. 85, no. 10, pp. 2072-2100, 2015.

[18] V. K. Sharma, S. K. Singh, U. Singh and V. Agiwal, "The inverse lindley distribution: A stress-strength reliability model with application to head and neck cancer data," Journal of Industrial and Production Engineering, vol. 32, no. 3, pp. 162-173, 2015.

[19] V. K. Sharma, S. K. Singh, U. Singh and F. Merovci, "The generalized inverse lindley distribution: A new inverse statistical model for the study of upside-down bathtub data," Communications in Statistics-Theory and Methods, vol. 45, no. 19, pp. 5709-5729, 2016.

[20] S. H. Alkarni, "Extended inverse lindley distribution: Properties and application," Springer Plus, vol. 4, pp. 690, 2015.

[21] K. V. P. Barco, J. Mazucheli and V. Janeiro, "The inverse power lindley distribution," Communications in Statistics-Simulation and Computation, vol. 46, no. 8, pp. 6308-6323, 2017.

[22] M. Eltehiwy, "Logarithmic inverse lindley distribution: Model, properties and applications," Journal of King Saud University-Science, vol. 32, no. 1, pp. 136-144, 2020.

[23] H. L. MacGillivray, "Skewness and asymmetry: Measures and orderings," the Annals of Statistics, vol. 14, no. 3, pp. 994-1011, 1986.

[24] A. L. Bowley, Elements of Statistics, London: P.S. King and Son, 1901.

[25] J. Moors, "A quantile alternative for kurtosis," Journal of the Royal Statistical Society. Series D (The Statistician), vol. 562, no. 37, pp. 25-32, 1988.

[26] J. A. Bishop, J. P. Formby and W. J. Smith, "Lorenz dominance and welfare: Changes in the U.S. distribution of income, 1967-1986." The Review of Economics and Statistics, vol. 73, no. 1, pp. 134-39, 1991.

[27] L. A. Prendergast and R. G. Staudte, "Quantile versions of the lorenz curve," Electronic Journal of Statistics, vol. 10, no. 2, pp. 1896-1926, 2016.

[28] C. D. Lai and M. Xie, Stochastic Ageing and Dependence for Reliability, New York: Springer, 2006.

[29] W. H. Alven, Reliability Engineering, ARINC. New Jersey: Prentice-hall, 1964. 